\title{
Swing Up and Balancing Implementation for The Pendubot Using Advanced Sliding Mode Control
}

\author{
Cao Van Kien, Nguyen Ngoc Son, Ho Pham Huy Anh \\ FEEE, DCSELAB, HCM City University of Technology, VNU-HCM, VietNam
}

\begin{abstract}
In this paper, an advanced sliding mode control (ASMC) with integral sliding function is proposed for the pendubot system which is a planar two degree of freedom (2DOF) robotic arm in the vertical plane with an actuator at the shoulder, but no actuator at the elbow. Using MATLAB/SIMULINK environment, the pendubot dynamics is implemented and the proposed ASMC control is applied for swing-up and balancing tasks the pendubot as to well follow with various trajectories. Simulation results demonstrate the performance and effectiveness of the proposed ASMC control in comparison with conventional sliding mode control applied in swing-up and balancing scheme of the pendubot system.
\end{abstract}

Keywords-pendubot; advanced sliding mode control; swing up; balancing scheme

\section{INTRODUCTION}

The problem of finding efficient control laws for underactuated systems has attracted a growing attention since these systems are featured by the fact that they possess fewer actuators than the DOFs to be controlled. Thus the underactuated mechanical systems have fewer control inputs than DOF and arise in lots of applications, such as aerospace and undersea robots. Since there exist tight couplings between actuated and underactuated DOFs in such systems, the control input isn't able to accelerate the state of the system in arbitrary directions. Consequently, underactuated mechanical plans cannot be controlled to follow arbitrary trajectories [1-2].

Recently many control researchers have concerned such control problems for underactuated mechanical systems in which including pendubot. Zhao and $\mathrm{Yi}$ in [3] proposed a GA-tuned bang-bang controller to successfully swing up the pendubot. $\mathrm{Ma}$ and $\mathrm{Su}$ in [4] introduced a new fuzzy approach for swing up control of pendubot. Authors in [5-6] applied versatile advanced nonlinear methods for swing up and balancing control of pendubot. The drawback of these results is that the proposed methods cannot satisfactorily control both of swing up phase and balancing phase of pendubot control process.

Sliding mode control based on the theory of variable structure systems is a robust method to control nonlinear uncertain systems in which including pendubot [7]. SerranoHeredia in [8] introduced the state feedback sliding mode to control the swing up of pendubot. Authors in [10-12] applied state feedback control, hybrid control and adaptive fuzzy PID control to stable control the swing up of pendubot. Recently, based on a sliding mode installed from the initial time, the system will be more robust against uncertainties than the existing sliding mode control systems with reaching phase. Hence an integral sliding mode has introduced to get the benefit such that the uncertain system behavior is eventually predictable from the initial time [7], [9], [13]. The disadvantage of these researches related to the fact that the control laws of proposed sliding mode controllers in these studies have not yet simultaneously successfully controlled both phases of swing-up and balance of the pendubot system.

In order to overcome this drawback, in this paper, an advanced sliding mode controller (ASMC) is proposed to successfully control both of swing-up and balancing phase of the pendubot system which is a planar two-link robotic arm in the vertical plane with an actuator at the shoulder, but no actuator at the elbow. Furthermore, the control laws of sliding surface have been improvingly chosen as to obtain the chattering-free motion.

\section{PENDUBOT MODELING}

The pendubot system structure is presented in Figure This pendubot system represents a planar two degree of freedom (2-DOF) robotic arm in the vertical plane with an actuator at the shoulder and no actuator at the below.

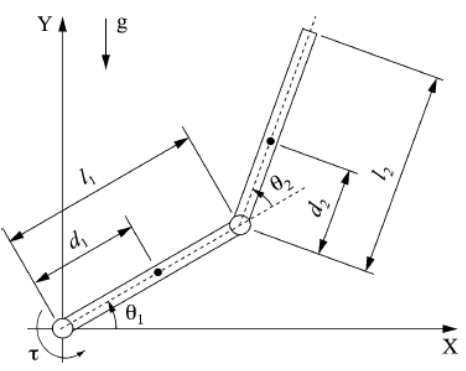

FIGURE 1. PENDUBOT SYSTEM.

Then the dynamic equations of pendubot system were expressed as follows

$$
\begin{gathered}
M(\theta) \ddot{\theta}+\mathrm{V}(\theta, \dot{\theta}) \dot{\theta}+G(\theta)=\left[\begin{array}{c}
\tau-b_{1} \dot{\theta}_{1} \\
-b_{2} \dot{\theta}_{2}
\end{array}\right] \\
M(\theta)=\left[\begin{array}{ll}
M_{11} & M_{12} \\
M_{21} & M_{22}
\end{array}\right]=\left[\begin{array}{cc}
P_{1}+P_{2}+2 P_{3} \cos \left(\theta_{2}\right) & P_{2}+P_{3} \cos \left(\theta_{2}\right) \\
P_{2}+P_{3} \cos \left(\theta_{2}\right) & P_{2}
\end{array}\right] \\
V(\theta, \dot{\theta})=\left[\begin{array}{ll}
V_{11} & V_{12} \\
V_{21} & V_{22}
\end{array}\right]=\left[\begin{array}{cc}
-P_{3} \dot{\theta}_{2} \sin \left(\theta_{2}\right) & -P_{3}\left(\dot{\theta}_{1}+\dot{\theta}_{2}\right) \sin \left(\theta_{2}\right) \\
P_{3} \dot{\theta}_{1} \sin \left(\theta_{2}\right) & 0
\end{array}\right] \\
G(\theta)=\left[\begin{array}{l}
G_{11} \\
G_{12}
\end{array}\right]=\left[\begin{array}{c}
P_{4} \cos \left(\theta_{1}\right)+P_{5} \cos \left(\theta_{1}+\theta_{2}\right) \\
P_{5} \cos \left(\theta_{1}+\theta_{2}\right)
\end{array}\right]
\end{gathered}
$$

With

$$
\begin{gathered}
P_{1}=m_{1} d_{1}^{2}+m_{2} l_{1}^{2}+l_{1}, P_{2}=m_{2} d_{2}^{2}+I_{2}, P_{3}=m_{2} l_{1} d_{2} \\
P_{4}=\left(m_{1} d_{1}+m_{2} l_{1}\right) g, P_{5}=m_{2} d_{2} g
\end{gathered}
$$

Given

$$
\begin{gathered}
H_{1}=-b_{1} \dot{\theta}_{1}-G_{11}-V_{11} \dot{\theta}_{1}-V_{12} \dot{\theta}_{2} \\
H_{2}=-b_{2} \dot{\theta}_{2}-G_{21}-V_{21} \dot{\theta}_{1}
\end{gathered}
$$




$$
\ddot{\theta}_{1}=\frac{\left(H_{1}+\tau\right) M_{22}-H_{2} M_{12}}{M_{11} M_{22}-M_{12} M_{21}}, \ddot{\theta}_{1}=\frac{H_{2} M_{11}-\left(H_{1}+\tau\right) M_{21}}{M_{11} M_{22}-M_{12} M_{21}}
$$

Given

with

$$
\ddot{\theta}_{1}=f_{1}+g_{1} \tau, \ddot{\theta}_{2}=f_{2}+g_{2} \tau
$$

$$
\begin{aligned}
& f_{1}=\frac{H_{1} M_{22}-H_{2} M_{12}}{M_{11} M_{22}-M_{12} M_{21}}, f_{2}=\frac{H_{2} M_{11}-H_{1} M_{21}}{M_{11} M_{22}-M_{12} M_{21}} \\
& g_{1}=\frac{M_{22}}{M_{11} M_{22}-M_{12} M_{21}}, g_{2}=\frac{-M_{21}}{M_{11} M_{22}-M_{12} M_{21}}
\end{aligned}
$$

Thus the state space equations are able to be derived. Given,

$$
x_{1}=\theta_{1}-\frac{\pi}{2}, x_{2}=\dot{x}_{1}, x_{3}=\theta_{2}, x_{4}=\dot{x}_{3}
$$

We have

$$
\dot{x}_{1}=x_{2}, \dot{x}_{2}=f_{1}+g_{1} \tau, \dot{x}_{3}=x_{4}, \dot{x}_{4}=f_{2}+g_{2} \tau
$$

Specific balancing positions,

Case of $\theta_{1}=\frac{\pi}{2}, \theta_{2}=0$ : both two links upright, $\theta_{1}=\frac{\pi}{2}$, $\theta_{2}=\pi: 1^{\text {st }}$ link upright and $2^{\text {nd }}$ link downright, $\theta_{1}=-\frac{\pi}{2}$, $\theta_{2}=0$ : both two links downright, $\theta_{1}=-\frac{\pi}{2}, \theta_{2}=\pi:$ the $1^{\text {st }}$ link downright and the $2^{\text {nd }}$ link upright.

\section{PROPOSED ASMC CONTROL IMPLEMENTATION}

\section{A. Pendubot Swing-Up Using SMC Control}

The error e is defined as follows

$$
\begin{aligned}
& e=x_{1}+x_{3} \\
& \dot{e}=\dot{x}_{1}+\dot{x}_{3}
\end{aligned}
$$

Construct the sliding surface

$$
s=c e+\dot{e}=c\left(x_{1}+x_{3}\right)+\left(x_{2}+x_{4}\right)
$$

In which $\mathrm{c}$ is a positive constant

$$
\begin{gathered}
s=c \dot{e}+\ddot{e}=c\left(x_{2}+x_{4}\right)+f_{1}+f_{2}+\left(g_{1}+g_{2}\right) \tau \\
\dot{s}=-K \operatorname{sign}(s)
\end{gathered}
$$

and $\mathrm{K}$ is also a positive constant

$$
\tau=\frac{-K \operatorname{sign}(s)-c x_{2}-c x_{4}-f_{1}-f_{2}}{g_{1}+g_{2}}
$$

Control law makes the sliding surface obtain $s \rightarrow 0$. Then the 2nd link upright, meanwhile the 1 st link uncontrolled. The speed will depend on the constant $\mathrm{c}$, with the bigger $\mathrm{c}$ then the faster of swing up with bounds determined.

Equation (1) shows that with error goes to 0 then the 2nd link gets upright in parallel with the gravity direction.

We choose the sliding surface s (3) when $s=0, t \rightarrow \infty$ then $e \rightarrow 0$.

This statement requires $\dot{s}$ having opposite sign with $\mathrm{s}$ and if $s=0$ then $\dot{s}=0$. In this condition, $\dot{s}_{\text {will be chosen as }}$ (4).
From (3), (4), the control law (5) will be induced.

\section{B. Proposed Advanced Sliding Mode Control}

The error e is now defined as follows

$$
\begin{gathered}
e=x_{1}+x_{3}-r \\
\dot{e}=\dot{x}_{1}+\dot{x}_{3}-\dot{r} \\
r=\alpha\left(r e f-\theta_{1}\right)
\end{gathered}
$$

Construct the sliding surface

$s=c e+\dot{e}=c\left(x_{1}+x_{3}-r\right)+\left(x_{2}+x_{4}-\dot{r}\right)$

In which $\mathrm{c}$ is a positive constant

$$
\begin{gathered}
\dot{s}=c \dot{e}+\ddot{e}=c\left(x_{2}+x_{4}-\dot{r}\right)-\ddot{r}+f_{1}+f_{2}+\left(g_{1}+g_{2}\right) \tau \\
\dot{s}=-K \operatorname{sigh}(s)
\end{gathered}
$$

and $\mathrm{K}$ is also a positive constant.

$$
\tau=\frac{-K \operatorname{sign}(s)-c x_{2}-c x_{4}+c \dot{r}+\ddot{r}-f_{1}-f_{2}}{g_{1}+g_{2}}
$$

ris signal for controlling $\theta_{1}$, coefficient $\alpha$ is chosen small enough to guarantee the stability of system.

From equation (6), new component $r$ is added in order to establish the new dynamic stability (keep balancing during moving) from the old static stability (balancing in static position). Responding sliding surface $s$ changes from (3) to (9). Using the same procedure, sliding surface now moves to 0 based on Equations (10) and (11). Given $r$ as a constant, there are two cases in steady state:

1. $\mathrm{r}$ and $\dot{\theta}_{1}$ possess the same sign, if $\mathrm{r}>0$ then $\theta_{1}$ increases:

Put $r=\alpha\left(r e f-\theta_{1}\right)(8)$, then $\alpha>0$, there is an error between ref and $\theta_{1}$, thus it will induce a value of $r$ help decreasing this error.

2. $\mathrm{r}$ and $\dot{\theta}_{1}$ possess the opposite sign, if $\mathrm{r}>0$ then $\dot{\theta}_{1}$ decreases:

Put $r=\alpha\left(r e f-\theta_{1}\right)(8)$, then $\alpha<0$, hence there is an error between ref and $\theta_{1}$, thus it will induce a value of $r$ help decreasing this error.

\section{Stability Of Advanced Sliding Mode Control}

Based on Lyapunov stability criteria, control rule is chosen as to satisfy s and $\dot{S}$ possess opposite signs. This statement keeps $s \rightarrow 0$. Then the sliding surface $s=c e+\dot{e}=0$ has the solution $e=C_{0} e^{-c t}$.

We see that with $t \rightarrow \infty, e \rightarrow 0$. Using equation (11), with $e \rightarrow 0$, the 2 nd link is in upright position and then $\dot{e}=0$. It means that the pendubot system is in static position.

In case of the proposed ASMC control, the control rule is added with component $r$ (equation (6)). Using equation (11), it will leads $s \rightarrow 0$ and then $e \rightarrow 0$. In steady state position, we have $x_{1} \neq-x_{3}$ and $x_{2}, x_{4} \neq 0$, and the bigger $r$ is then the more the difference possesses. With $\mathrm{r}$ is chosen as (8), $\mathrm{r}$ can be easily modified via $\alpha$ and $\theta_{1}$. It means that $\theta_{1}$ will converge to ref value with small enough. The stability 
condition of proposed ASMC applied in balancing control of the pendubot system has been demonstrated.

\section{SIMULATION RESULTS}

The simulation will be realized with following parametric values

$$
\begin{gathered}
m_{1}=0.2 \mathrm{~kg}, m_{2}=0.1 \mathrm{~kg}, l_{1}=l_{2}=0.2 \mathrm{~m}, b_{1}=b_{2}=0.01 \\
d_{1}=\frac{1}{2} l_{1}, d_{2}=\frac{1}{2} l_{2}, I_{1}=\frac{1}{3} m_{1} l_{1}^{2}, I_{2}=\frac{1}{3} m_{2} l_{2}^{2}
\end{gathered}
$$

Fig. 2 represent the Simulink simulation block scheme with Sliding mode block and pendubot block has been presented above.

The excellent simulation results confirm the performance and the effectiveness of the proposed ASMC control in comparison with conventional sliding mode control applied in balancing scheme of the pendubot system.

Firstly, Fig. 3 represents the good swing up response of pendubot system using the SMC control. In this test, the corresponding referential values are of the case of

$\theta_{1}=\frac{\pi}{2}, \theta_{2}=0$, in which both of links in upright positions.

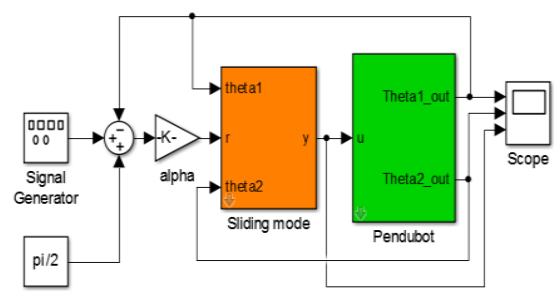

FIGURE. 2. SIMULINK SIMULATION BLOCK SCHEME.
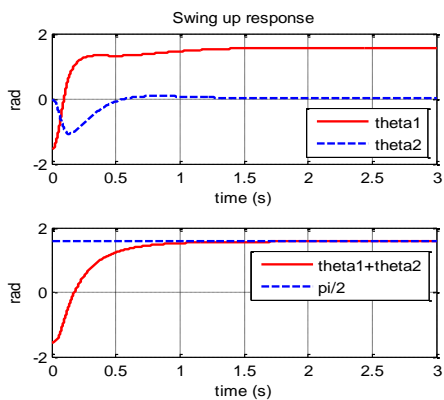

FIGURE 3. SWING UP RESPONSE OF PENDUBOT SYSTEM.
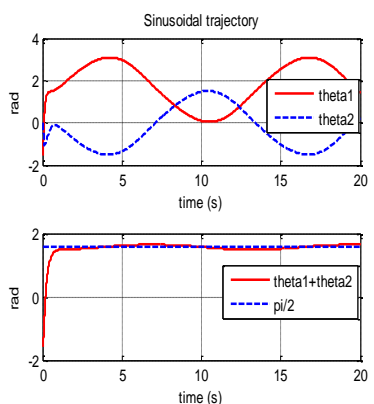

FIGURE 4. BALANCING CONTROL WITH SINUSOIDAL TRAJECTORY
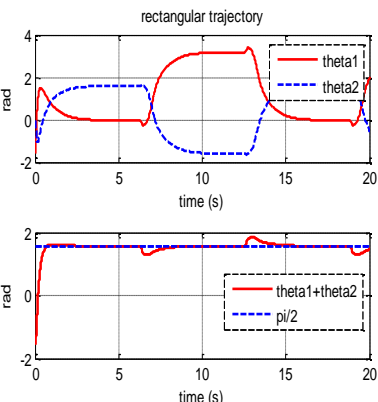

FIGURE 5. BALANCING CONTROL WITH RECTANGULAR TRAJECTORY.
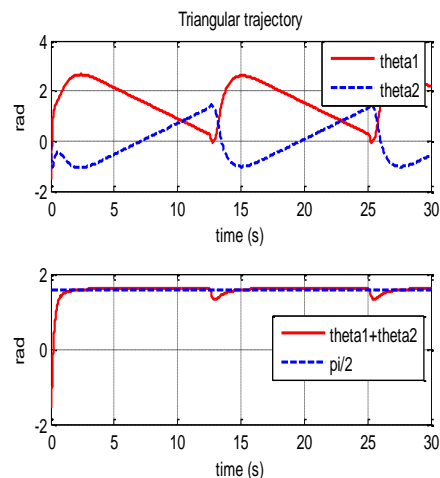

\section{FIGURE. 6. BALANCING CONTROL WITH TRIANGULAR} TRAJECTORY.

Fig. 4, Fig. 5, Fig. 6 and Fig. 7 represent the very good response of the proposed ASMC control applied to the pendubot system which ensure the performance and efficiency in balancing control the pendubot system with sinusoidal, rectangular and triangular trajectories, respectively.

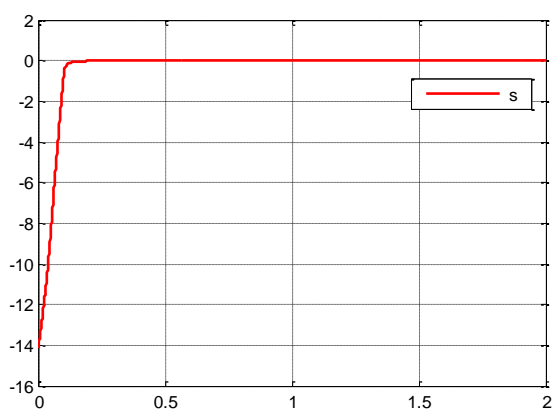

FIGURE. 7. SLIDING SURFACE S WITH R BEING CONTINUOUS DERIVATIVE.

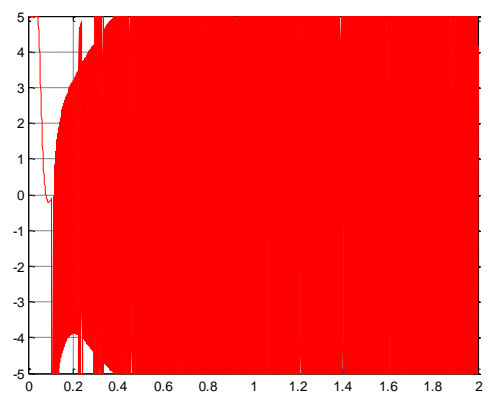

FIGURE. 8. OUTPUT OF ASMC CONTROL WITH SIGN EQUATION. 


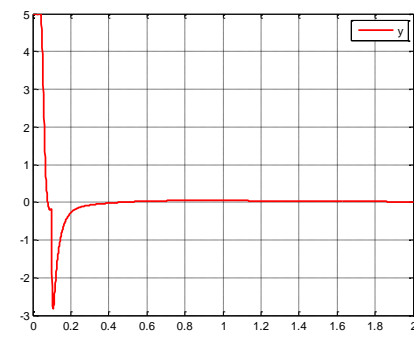

FIGURE. 9. OUTPUT OF ASMC CONTROL WITH SAT.EQUATION.

Furthermore, in order to eliminating the chattering phenomenon, we applied the saturation equation instead of the classic K-sign.

In detail, with $\dot{s}=-K \operatorname{sign}(s)$, the strongly chattering phenomenon will appear as illustrated in Fig.8. By replacing the sign equation with the saturation equation. The chattering phenomenon is completely eliminated as represented in Fig. 9.

\section{CONCLUSIONS}

This paper concerns the strong stabilization for a typical example of underactuated robots called the pendubot. This paper showed the design method of a novel strongly stabilizing controller for the upright equilibrium point of the pendubot system by implementing a specific output and employing the advanced sliding mode control. The simulation results were investigated to validate the obtained theoretical results. The paper introduced the new good features of the swing-up and balancing implementation for the pendubot system using the proposed advanced sliding mode control.

\section{ACKNOWLEDGEMENTS}

This paper was supported by NAFOSTED and VNUHCM, Viet Nam.

\section{REFERENCES}

[1] Mingjun Z., Tzyh-Jong T., Hybrid Control of the Pendubot, IEEE/ASME Transactions on Mechatronics, vol. 7, no. 1, pp. 79-86, 2002 .

[2] WeiLi , Kazuo Tanaka, and Hua O. Wang, Acrobatic Control of a Pendubot, IEEE Transactions on Fuzzy Systems, vol. 12, no. 4, pp. 549-552, 2004

[3] Dongbin Zhao and Jianqiang Yi, Swing up Pendubot with a GA-tuned Bang-bang Controller, Proceedings of the 2003 IEEE Intemational Conf. on Robotics, Jntelligent Systems and Signal Processing, pp. 752-757, 2003

[4] X. Q. Ma and C.Y. Su, A New Fuzzy Approach for Swing up Control of Pendubot, Proc. of American Control Conf. Anchorage, pp. 1001-6, 2002.

[5] Xin Xin and Kousuke Juuri, Existence and Design of a Strongly Stabilizing Controller for the Pendubot, SICE Annual Conf. 2012, pp. 1598-1602, 2012

[6] T.Albahkali,T.Das, Swing-up Control of Pendubot: An ImpulseMomentum Approach, IEEE Trans. on Robotics, vol. 25, no. 4, pp. 975-982, 2009.

[7] D. Qian, J. Yi, and D. Zhao, Hierarchical Sliding Mode Control to Swing up a Pendubot, Proc. of the American Control Conf., pp. 52545259, 2007

[8] J.S.Heredia, A.G. Loukianov, E.B.Corrochano, Sliding Mode Block Control Regulation of the Pendubot, Proc. of The 50th IEEE Conf. on Decision and Control and European Control Conf. (CDC-ECC), pp. 8249-54, 2011.
[9] Dong Sang Yoo, 2013, Balancing Control for the Pendubot using Sliding Mode, 44th International Symposium on Robotics (ISR), pp. 14, Oct. 2013.

[10] J. Serrano-Heredia, A.G. Loukianov, E.B.Corrochano, State Feedback Block Control Regulation of Pendubot, Electrical Engineering Computing Science and Automatic Control (CCE) $8^{\text {th }}$ International Conf., pp. 1-6, 2011.

[11] Rowland W. O'Flaherty, Ricardo G. Sanfelice and Andrew R. Teel, Hybrid Control Strategy for Robust Global Swing-Up of the Pendubot, 2008 American Control Conf., , pp. 1424-1429, 2008.

[12] C.J. Wu, T.L. Lee, Yu-Yi Fu and L.C. Lai, Auto-Tuning Fuzzy PID Control of a Pendubot System, Proc. of Inter. Conf. on Mechatronics, pp. 1-6, 2007 .

[13] H. Jian, D. Feng and W. Yongji, Sliding Mode Control with Nonlinear Disturbance Observer for a Class of Underactuated System, Proc. of the 32nd Chinese Control Conf. pp. 541-546, 2013 